\title{
A novel chemometric classification for FTIR spectra of mycotoxin-contaminated maize and peanuts at regulatory limits
}

\author{
Gregor Kos ${ }^{a}$, Markus Sieger ${ }^{b}$, David McMullin', Celine Zahradnikc, Michael Sulyokc, Tuba Öner ${ }^{b}$, \\ Boris Mizaikoff (10 ${ }^{\mathrm{b}}$ and Rudolf $\mathrm{Krska}^{\mathrm{c}}$ \\ 5 aDepartment of Atmospheric and Oceanic Sciences, McGill University, Montreal, QC, Canada; 'bnstitute of Analytical and Bioanalytical \\ Chemistry, Ulm University, Ulm, Germany; 'Department of Agrobiotechnology (IFA-Tulln), University of Natural Resources and Life Sciences, \\ Tulln, Austria
}

\section{ABSTRACT}

The rapid identification of mycotoxins such as deoxynivalenol and aflatoxin $B_{1}$ in agricultural commodities is an ongoing concern for food importers and processors. While sophisticated chromatography-based methods are well established for regulatory testing by food safety authorities, few techniques exist to provide a rapid assessment for traders. This study advances the development of a mid-infrared spectroscopic method, recording spectra with little sample preparation. Spectral data were classified using a bootstrap-aggregated (bagged) decision tree method, evaluating the protein and carbohydrate absorption regions of the spectrum. The method was able to classify $79 \%$ of 110 maize samples at the European Union regulatory limit for deoxynivalenol of $1750 \mu \mathrm{g} \mathrm{kg}{ }^{-1}$ and, for the first time, $77 \%$ of 92 peanut samples at $8 \mu \mathrm{gg}^{-1}$ of aflatoxin $B_{1}$. A subset model revealed a dependency on variety and type of fungal infection. The employed CRC and SBL maize varieties could be pooled in the model with a reduction of classification accuracy from $90 \%$ to $79 \%$. Samples infected with Fusarium verticillioides were removed, leaving samples infected with $F$. graminearum and $F$. culmorum in the dataset improving classification accuracy from $73 \%$ to $79 \%$. A $500 \mu \mathrm{g} \mathrm{kg}^{-1}$ classification threshold for deoxynivalenol in maize performed even better with $85 \%$ accuracy. This is assumed to be due to a larger number of samples around the threshold increasing representativity. Comparison with established principal component analysis classification, which consistently showed overlapping clusters, confirmed the superior performance of bagged decision tree classification.

\section{ARTICLE HISTORY}

Received 31 March 2016

Accepted 17 July 2016

\section{KEYWORDS}

Analysis - MIR;

chemometrics; aflatoxins;

trichothecenes; cereals;

peanuts

\section{Introduction}

Mycotoxins are secondary metabolites produced by fungal species that infest agricultural commodities such as peanuts, maize and wheat. Globally, mycotoxins are a major driver behind spoiled food with important implications for human and animal health, causing significant financial damage ( $\mathrm{Wu}$ 2007). The global economic cost due to mycotoxins is estimated to run to billions of US dollars (Swamy et al. 2004). Vardon et al. (2003) estimated an annual range of losses from US $\$ 0.5$ million to over US $\$ 1.5$ billion from aflatoxin in maize and peanuts, fumonisins in maize, and deoxynivalenol in wheat. Typically, plants are infected by mycotoxigenic fungi, e.g., during particularly wet growth seasons or during storage, if moisture conditions greater than $14 \%$ prevail (Richard 2007) and support fungal growth. Mycotoxigenic fungi can be plant pathogens, such as Fusarium graminearum infecting wheat and maize. $F$. verticillioides and Aspergillus flavus will produce mycotoxins on senescent or stressed maize crops. Soil-borne fungi may occur on developing kernels and later produce mycotoxins in storage if conditions permit (Miller 2008). Resulting concentrations in localised 'hot-spots' can be so high that they could render an entire crop batch unsuitable for consumption (Choudhary \& Kumari 2010).

Aflatoxins and deoxynivalenol are among the most important toxic fungal metabolites, because of their global occurrence and toxicity. Aflatoxins are mainly produced by $A$. flavus, $A$. parasiticus and A. nomius (Ertas et al. 2011). Aflatoxin $B_{1}$ is classified as a Group 1 human carcinogen by the IARC (1993) (Smela et al. 2001) and is frequently detected in peanuts and peanut-based food and feed originating from sub-tropical and tropical regions. However, aflatoxins have recently 
been detected in European maize harvested after a summer with similarly hot conditions, which are increasingly linked to climate change (Prandini et al. 2009). Aflatoxins are a cause of human liver cancer and, in high doses, have caused deaths from aflatoxicosis. Significant negative effects of aflatoxin on child growth have been reported, as well as immune modulation (Groopman et al. 1988; IARC 2016).

Deoxynivalenol belongs to the group of B-trichothecenes and is mainly produced by F. graminearum and F. culmorum. Just like aflatoxins, it is a mycotoxin of global concern, occurring at sometimes high to very high concentrations in the $\mathrm{mg} \mathrm{kg}^{-1}$ range. Affected commodities are wheat, barley and oats, and maize. Thus it becomes important as a contaminant of food despite its relatively low toxicity. In animals, particularly swine, deoxynivalenol-containing feed starting at levels of $1 \mu \mathrm{g} \mathrm{kg}^{-1}$ as reported by Richard (2007) leads to feed refusal, vomiting and intestinal damage, especially to the kidneys (Pestka 2010).

Because of their global occurrence, legislation for both aflatoxins and deoxynivalenol with other Fusarium toxins has been enacted to ensure food and feed chain safety. A summary of European Union (EU) regulatory limits and non-binding guidance values (e.g., for deoxynivalenol in animal feed) regarding aflatoxin $B_{1}$ and deoxynivalenol are shown in Table 1. For

90 analytical screening methods to be developed, these limits represent an upper limit regarding classification performance.

Due to increased global trade of agricultural commodities, an expanding number of extreme weather events and climate change-related processes, there is a strong need to increase testing during import, storage

Table 1. European Union limits and guidance values $\left(\mu \mathrm{g} \mathrm{kg}^{-1}\right)$ for aflatoxin $B_{1}$ and deoxynivalenol in food and animal feed.

\begin{tabular}{|c|c|c|}
\hline Commodity & Aflatoxin $B_{1}$ & Deoxynivalenol \\
\hline \multicolumn{3}{|l|}{ Food } \\
\hline Peanuts (for consumption) & 2 & \\
\hline Peanuts (for processing) & 8 & \\
\hline Cereals for human consumption & 2 & 750 \\
\hline Cereal-based baby food & 0.1 & 200 \\
\hline Bread and cereal snacks & & 500 \\
\hline Maize (unprocessed) & & 1750 \\
\hline Maize (milling fraction $<500 \mu \mathrm{m}$ ) & n.a. & 1250 \\
\hline Maize (milling fraction $>500 \mu \mathrm{m}$ ) & n.a. & 750 \\
\hline \multicolumn{3}{|l|}{ Animal feed } \\
\hline Feed (except below) & 20 & 8000 (cereals) \\
\hline Maize by-products & & 12,000 \\
\hline Calves and lamb feed & 10 & 2000 \\
\hline Dairy feed & 5 & \\
\hline Pig feed & 20 & 900 \\
\hline
\end{tabular}

and processing. Current state-of-the-art methodology relies mostly on LC-MS (e.g., Malachová et al. 2014), HPLC-UV and HPLC-FLD (e.g., Rodrigues et al. 2011) for the determination of toxin concentrations. Especially, LC-MS methods are highly sensitive and have been comprehensively validated (e.g., by Malachová et al. 2015) to provide documented accuracy and precision for regulatory purposes. Analyses require advanced laboratory facilities and highly qualified personnel, thus making these methods difficult and costly to use for 'informal' tests by importers, traders, and food- and feed-processing companies. One exception is ELISA (e.g., Turner et al. 2009), which has found application outside regulatory testing, but with ongoing issues regarding cross-reactivities and the need to handle solvents and testing solutions. A review about ELISA and similar assay-based methods was recently published by Chauhan et al. (2016).

Spectroscopy-based rapid screening methods have the potential to fill this gap regarding much-requested 'informal' testing methods. Rather than replacing LCMS and similar methods, these complementary screening methods provide importers and processors with suitable easy-to-use screening tools without the need for extensive laboratory facilities and highly qualified personnel. Within the EU, costs for sampling and analysis are typically borne by the importer according to Commission Regulation (EC) No. 1152/2009. Therefore, preliminary analyses conducted with rapid screening methods could provide traders with information about potential contamination. By removing contaminated batches right away, the number of samples submitted to regulatory testing can be reduced. As a consequence, rapid methods are suitable to achieve goals of increased testing at, for example, ports of entry and reduce the number of regulatory testing procedures. A large number of simple on-site checks during import, storage and processing, therefore, becomes feasible.

Infrared (IR) spectroscopy-based methods provide simple, non-destructive means to obtain information about a sample. Especially, the near-infrared range (NIR; 13,500 to $4000 \mathrm{~cm}^{-1}$ ), with its inherent advantages of being insensitive to moisture and transparency of glass containers, greatly simplifies the measurement process. NIR spectroscopy has been successful to measure protein and moisture content as a means to assess grain quality (e.g., by Maertens et al. 2004). While NIR provides overtone frequencies of molecule and functional group vibrations, these are directly accessible using the mid-infrared range (MIR; 4500 to $400 \mathrm{~cm}^{-1}$ ). Typical MIR absorption frequencies recorded of cereal samples include hydroxyl, methylene, carbonyl (CO) stretching, 
$150 \mathrm{NH}$ bending and $\mathrm{CO}$ stretching vibrations (Kos et al. 2003). The latter (CO and NH vibrations) can further be related to protein and carbohydrates present in the sample. Together with water bands, these spectral features dominate the IR spectrum of foods. Additionally, 155 the fingerprint region of the spectrum between 900 and $400 \mathrm{~cm}^{-1}$ provides unique information about the sample, although assignment to common vibrations is possible only in selected cases. Changes due to fungal contamination result in changes due to protein and 160 carbohydrate content, i.e., changes to $\mathrm{CO}$ and $\mathrm{NH}$ bands, which allow for indirect detection of fungi and their toxins. A summary of state-of-the-art IR spectroscopy-based rapid methods was provided by McMullin et al. (2015). Recent advances in IR source technology

165 with the availability of MIR quantum cascade lasers will significantly boost source power, which is expected to lead to improved signal-to-noise ratios and, therefore, higher sensitivity (Young et al. 2009).

Protein and carbohydrate changes due to fungal 170 contamination result in small changes to the MIR spectrum Kos et al. (2003). Interpretation of the generated IR spectrum is challenging, involving several small changes across the absorption spectrum in the protein and carbohydrate absorption region.

175 Evaluation of single (univariate) absorption band maxima, an approach traditionally used for classification and quantification, is not recommended. Rather, a multivariate strategy, also called 'chemometrics', where one or more spectral windows composed of

180 typically hundreds of wavelengths of overlapping bands are used simultaneously to build a classification or quantitation model, is used (Mark \& Workman 2010). While generally considered less intuitive, a wealth of information is provided and small changes to the selected spectral regions can be detected and used in the model. For a general introduction and review to chemometric methods used to assess food quality, see Karoui et al. (2010).

Among the most popular chemometric methods is 190 principal component analysis (PCA), which is used as a tool for data reduction and classification. The method builds a set of new orthogonal variables with maximised variance (loadings) from the original absorbance information and calculates a score for each sample.

195 Score plots starting with those explaining most of the variance reveal patterns and clusters of samples that can be used for classification. Among quantitative methods principal component regression (PCR), a combination of PCA, to remove correlations between 200 variables, and multilinear regression (MLR) are popular as is partial least squares regression (PLS) (Naes et al. 2002; Mark \& Workman 2010). KNN is an example of a non-parametric method that counts the number of closely spaced data points and identify its class (e.g., above or below a defined contamination threshold) in a training set and based on a majority vote assigns the class of an unknown (Mariey et al. 2001). Similarly, decision trees, 'flow-chart'-like models, can be built with each node testing a property of the data (Quinlan 1986). While these methods tend to create overfitted models (i.e., too closely matching the training set and leading to model instability), bootstrap aggregation (also known as 'bagging') combines data from randomly generated training trees. Variance from noise and error-prone data is minimised, providing a more stable classifier (Breiman 1996).

Multivariate methods have been used extensively to assess food quality and identify and quantify major constituents of foods. Examples are the assessment of protein content in wheat (Williams \& Sobering 1993), the determination of fat in milk samples (Inon et al. 2004), the pork content in chicken meat (Al-Jowder et al., 1997) and the classification of modified starches (Fernandez Pierna et al., 2005). Microorganisms such as lactic acid bacteria have also been identified using MIR spectroscopy (Amiel et al. 2000).

Among minor and trace constituents, MIR spectroscopy with chemometric data analysis plays an increasing role as an effective tool to screen for the presence and quantities of contaminants. Table 2 provides an overview of reports published using MIR methods and chemometrics for the determination of trace components in food and feed products.

The application of NIR spectroscopic methods dominates reports in the scientific literature, because of widespread use due to simple instrumentation. MIRbased techniques follow with the occasional use of Raman spectroscopy and photo-acoustic spectroscopy, the latter of which is quite suitable for single-kernel analysis.

Previous work was primarily focused on developmental aspects, often with relatively few samples in the dataset or large mycotoxin concentration differences in order to demonstrate the feasibility of the approach (Kos et al. 2003). Large concentrations also facilitated the initial identification of changes to the IR spectrum, related to changes in protein and carbohydrate concentration due to fungal infection. For application of these methods in real-life environments, it is necessary to have a large number of samples at relevant concentrations (e.g., at the regulatory limit) to reflect accurately the inherent variability of the dataset. To our knowledge, the detection of aflatoxins in peanuts at legal limits has not yet been attempted. Similarly, reports for classification of DON-contaminated maize in the

\section{5}


range of EU legal limits are not yet available. Furthermore, Table 2 shows that PCA and PLS techniques are highly popular for classification and quantification, respectively, compared with techniques such as KNN and decision trees. The latter have not yet been investigated and tested extensively.

In this study we present classification results for deoxynivalenol in maize and, for the first time at trace levels, aflatoxin $B_{1}$ in peanuts using MIR-ATR spectroscopy with chemometrics. Specifically, we compared results using PCA and, also a first, a bagged decision tree methodology for classification. Reference data were obtained employing a single, well-documented LC-MS method. Classification limits for both mycotoxins follow EU reg-

270 ulations, and a large number of samples were investigated at relevant concentration ranges to allow for full validation of the multivariate approach.

\section{Materials and methods}

\section{Sampling and sample preparation}

275 Maize samples were provided by Saatbau Linz (Linz, Austria; SBL) and the Cereal Research Centre (Szeged, Hungary; CRC). All samples were maize hybrids with dent or flint kernel types used for kernel production in the field. Either, natural infection, generally yielding 280 low mycotoxin contaminations, or artificial infection by silk injection or toothpick inoculation for high mycotoxin concentrations, with $F$. graminearum, $F$. verticillioides or F. culmorum was performed. All injection and inoculation work was done in the field before 285 harvest. Silk channel injection was performed 5 days after 50\% silking. Maize ears were injected with $2 \mathrm{ml}$
Fusarium suspension at a concentration of $5 \times 10^{5}$ conidia $\mathrm{ml}^{-1}$ in the silk channel. Fusarium conidial suspension was produced with the bubble breeding method in mung bean broth (Mesterházy 1977). Small aliquots were frozen at $-80^{\circ} \mathrm{C}$ and quickly thawed at $35^{\circ} \mathrm{C}$ before use. Inoculation with the toothpick method was performed 10 days after $50 \%$ silking. A hole was drilled in the centre of the ear, and a tooth pick overgrown with Fusarium isolate was inserted (Chungu et al. 1996). Harvested samples were milled (Romer, Union, MO, USA) and spectral analysis was carried out on the 100-250 $\mu \mathrm{m}$ sieve fraction (Retsch, Hahn, Germany).

For peanuts, 92 different, naturally infected samples from public markets in three different African countries (Tanzania, Mozambique and Burkina Faso) were collected. Each sample was analysed individually and no mixing was performed. Peanuts were manually ground to an oily paste with a mortar and pestle prior to analysis.

\section{Infrared data acquisition}

MIR spectra were generated with a portable Fourier transform IR spectrometer equipped with an attenuated total reflection (FTIR-ATR) unit (Bruker Alpha, Karlsruhe, Germany). A diamond crystal with one internal reflection served as an ATR element (Bruker, Platinum ATR). For improved portability an L-alanine doped triglycine sulfate (DLaTGS) detector was employed. Spectra were recorded in the MIR range (4000 to $575 \mathrm{~cm}^{-1}$ for maize; $475 \mathrm{~cm}^{-1}$ as the lower limit for peanuts) with a resolution of $4 \mathrm{~cm}^{-1}$. Spectra

Table 2. Infrared spectroscopic methods and chemometrics employed for the analysis of minor constituents in foods.

\begin{tabular}{|c|c|c|c|c|}
\hline Commodity & Parameter & Instrument & Chemometrics & Reference \\
\hline Sugar & Polysaccharides & MIR & PCA & Cerna et al. (2003) \\
\hline Maize & Deoxynivalenol & MIR-ATR & PCA & Kos et al. (2003) \\
\hline Legumes & Minerals & NIR-reflectance & MPLS & Cozzolino and Moron (2004) \\
\hline Wine & Phenolic compounds & NIR & PLS & Cozzolino et al. (2004) \\
\hline Wheat & Deoxynivalenol & MIR-ATR, DRIFT & PLS, MLR & Abramovic et al. (2007) \\
\hline Milk & Melamin & NIR, MIR-ATR, DRIFT & PLS & Mauer et al. (2009) \\
\hline Maize & Aflatoxin $B_{1}$ & NIR-reflectance & PLS & Fernandez-lbanez et al. (2009) \\
\hline Wheat & Deoxynivalenol & NIR & PLS & Firrao et al. (2010) \\
\hline Maize & Fumonisins & MIR & ANN & Draganova et al. (2010) \\
\hline Maize & Fusarium spp. & NIR-DRIFT & SIMCA, ANN & Vermeulen et al. (2012) \\
\hline Wheat & Ergot bodies & NIR & PLSDA, SVM & Williams et al. (2012) \\
\hline Maize & F. verticillioides & NIR & PCA & Sirisomboon et al. (2013) \\
\hline Rice & Aflatoxigenic fungi & NIR & PLS & De Girolamo et al. (2014) \\
\hline Wheat & Deoxynivalnol & NIR & LDA & Qiang et al. (2014) \\
\hline Rice & Aflatoxin $B_{1}$ & NIR & PLS & Lee et al. (2015) \\
\hline Maize & Aflatoxins & NIR, MIR, Raman & MLR, PCR, PLS & Lee and Herrman (2015) \\
\hline Maize & Fumonisins & SERS & KNN, LDA, PLS & Kaya-Celiker et al. (2016) \\
\hline Peanut seeds & Aspergillus spp. & PAS & LDA & \\
\hline
\end{tabular}

Note: ANN, artificial neural networks; ATR, attenuated total reflection; DRIFT, diffuse reflectance Fourier transform infrared spectroscopy; KNN, K-nearest neighbour classification; LDA, linear discriminant analysis; MIR, mid-infrared spectroscopy; MLR, multilinear regression; NIR, near-infrared spectroscopy; PAS, photo-acoustic spectroscopy; PCA, principal component analysis; (M)PLS, (modified) partial least squares regression; PLSDA, partial least squares discriminant analysis; SERS, surface-enhanced Raman spectroscopy; SIMCA, soft independent modelling of class analogy; SVM, support vector machines. 
were collected from 64 scans with a sample and reference gain of 1 . Phase correction was applied using a power spectrum with a phase resolution of 32 and Blackman-Harris 3-Term apodisation function. Solvents employed for cleaning were iso-propanol for maize and methanol, acetone and iso-propanol (applied sequentially) for peanuts.

Typically, 10 repeat measurements, each with a new subsample, were performed on the ground and sieved maize and ground peanut samples. While a spectral window from 1800 to $800 \mathrm{~cm}^{-1}$ was employed for chemometric modelling, the acquisition of the full
MIR range was feasible by using an FTIR instrument, maximising spectral information content for initial exploratory data analysis for the identification of suitable spectral windows. See Figures 1(a) and (b) for typical repeat spectral measurements without pretreatment. Carbohydrate and protein-related vibrations at 1000 and $1500 \mathrm{~cm}^{-1}$, respectively, are prominent spectral features. Upon fungal infestation, these bands are subject to changes due to changes in protein and carbohydrate content (Boyacioğlu \& Hettiarachchy 1995; Kos et al. 2003; McMullin et al. 2015). For the purpose of chemometric modelling, these changes were then

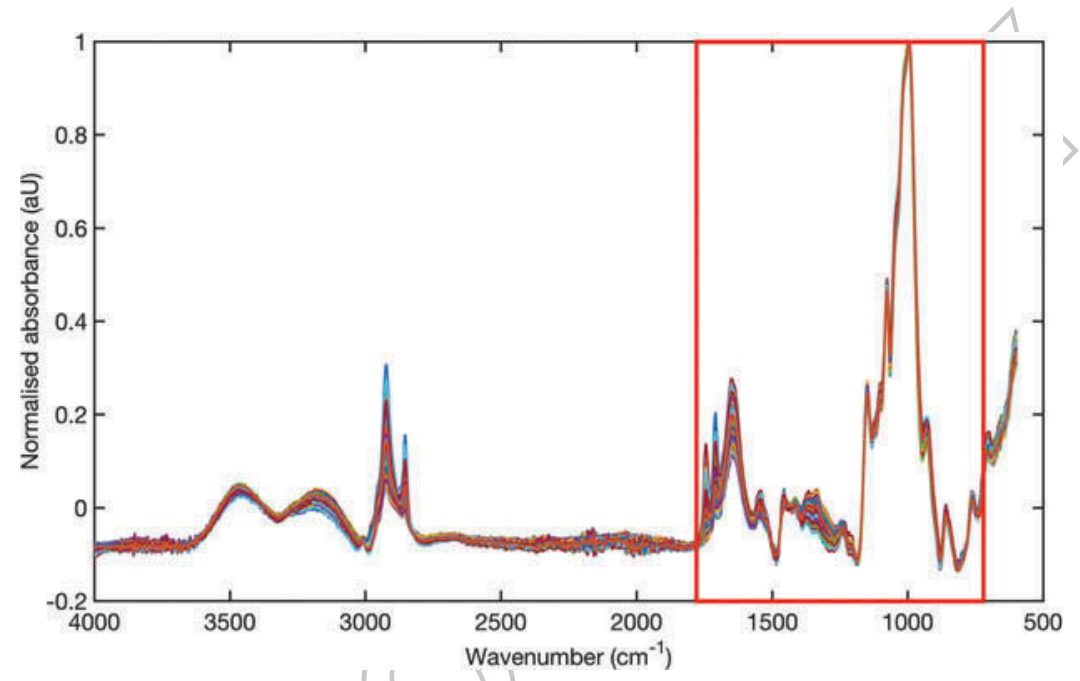

Figure 1(a). MIR spectra of maize; repeat measurements of 10 different subsamples. The (boxed) spectral window between 1800 and $800 \mathrm{~cm}^{-1}$ was used for chemometric modelling.

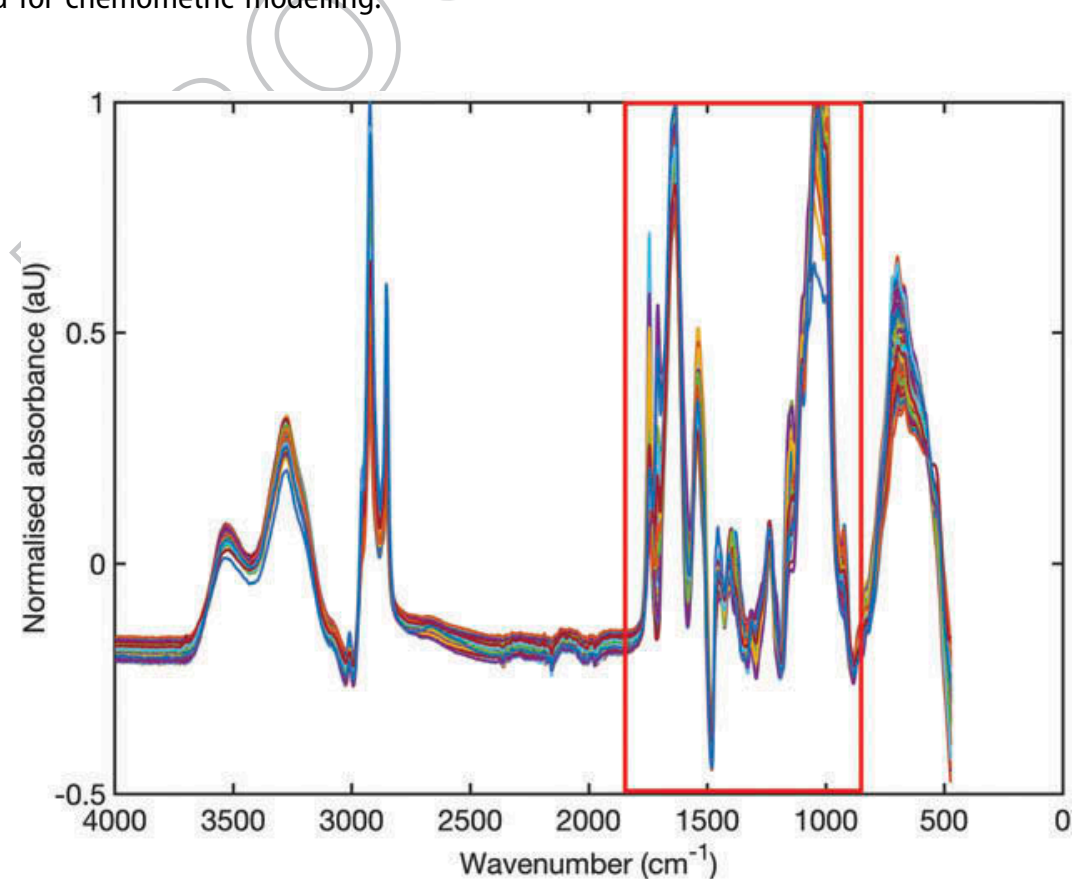

Figure 1(b). MIR spectra of peanuts; repeat measurements of 10 different subsamples. The (boxed) spectral window between 1800 and $800 \mathrm{~cm}^{-1}$ was used for chemometric modelling. 
related to toxin concentrations, since these are the regulated parameters and facilitate practical application of the developed model.

The less specific $\mathrm{CH}$ and $\mathrm{OH}$-related bands at approximately 2900 and $3400 \mathrm{~cm}^{-1}$ are also clearly visible, but were not used for data analysis for their lack of specificity (Kos et al. 2003). Band assignments for spectra are available in Table 3.

\section{LC-MS/MS reference measurements}

Homogenised samples of $5 \mathrm{~g}$ were extracted with $20 \mathrm{ml}$ of extraction solvent (acetonitrile/water/acetic acid $79: 20: 1, \mathrm{v} / \mathrm{v} / \mathrm{v}$ ) for $90 \mathrm{~min}$ on a rotary shaker (GFL 3017, Burgwedel, Germany). After a $1+1(\mathrm{v} / \mathrm{v})$ dilution using dilution solvent (acetonitrile/water/acetic acid 79:20:1, v/v/v), the diluted extracts were injected without further pretreatment.

The chromatographic method, chromatographic and mass spectrometric parameters are described by

360 Malachová et al. (2014). Briefly, LC-MS/MS screening of target microbial metabolites was performed with a QTrap 5500 LC-MS/MS System (Applied Biosystems, Foster City, CA, USA) equipped with TurboIonSpray electrospray ionisation (ESI) source and a 1290 Series HPLC System (Agilent, Waldbronn, Germany) Chromatographic separation was performed at $25^{\circ} \mathrm{C}$ on a Gemini C-18-column, $150 \times 4.6 \mathrm{~mm}$ i.d., $5 \mu \mathrm{m}$ particle size, equipped with a $\mathrm{C} 184 \times 3 \mathrm{~mm}$ i.d. security guard cartridge (Phenomenex, Torrance, CA, USA).

ESI-MS/MS was performed in the time-scheduled MRM mode both in positive and negative polarities in two separate chromatographic runs per sample by scanning two fragmentation reactions per analyte. The MRM detection window of each analyte was set to its expected retention time \pm 23 and $\pm 29 \mathrm{~s}$ in positive and negative modes, respectively. Confirmation of positive analyte identification was obtained by the acquisition of two MRMs per analyte (with the exception of

Table 3. Band assignments for MIR vibrations in maize and peanuts.

\begin{tabular}{lc}
\hline Vibration & Wave number $\left(\mathrm{cm}^{-1}\right)$ \\
\hline -CH trans stretching & 3025 \\
-CH cis stretching & 3006 \\
$\mathrm{CH}\left(-\mathrm{CH}_{2}-\right)$ asymmetric stretch & 2924 \\
$\mathrm{CH}\left(-\mathrm{CH}_{2}-\right)$ symmetric stretch & 2854 \\
$\mathrm{C}-\mathrm{O}$ ester stretching & 1745 \\
$\mathrm{C}-\mathrm{O}$ fatty acids & 1710 \\
Amide I & 1650 \\
Amide II & 1540 \\
$-\mathrm{C}-\mathrm{H}$ stretching of $\mathrm{CH}_{2}$ and $\mathrm{CH}_{3}$, fatty acids & $1490-1425$ \\
$-\mathrm{C}-\mathrm{H}$ symmetric bending methyl groups & $1410-1330$ \\
Ring vibrations of carbohydrates & 1030 \\
\hline
\end{tabular}

moniliformin which exhibited only one fragment ion). This yielded 4.0 identification points according to European Commission Decision 2002/657 (European Commission 2002). In addition, the LC retention time and the intensity ratio of the two MRM transitions agreed with the related values of an authentic standard within $0.1 \mathrm{~min}$ and $30 \%$ rel., respectively. The accuracy of the method is verified on a routine basis by regular participation in proficiency testing schemes (Malachová et al. 2015). The LODs were $1.2 \mu \mathrm{g} \mathrm{kg}^{-1}$ for deoxynivalenol and $0.24 \mu \mathrm{g} \mathrm{kg}^{-1}$ for aflatoxin $B_{1}$.

\section{Infrared data classification and validation}

These acquired IR and LC-MS/MS reference data formed the base for the modelling exercise presented here. Classification thresholds of 1750 and $500 \mu \mathrm{g} \mathrm{kg}^{-1}$ were chosen for deoxynivalenol in maize and $8 \mu \mathrm{g} \mathrm{kg}^{-1}$ for aflatoxin $B_{1}$ in peanuts in agreement with European Union legislation. Chemometric modelling and plotting was carried out using Matlab (version 2015a), including data import, pre-processing, spectral window selection, chemometric modelling and creation of spectral plots. Due to the heterogeneity of the maize samples provided with regard to multiple variables (e.g. yariety, fungal infection, etc.) (Table 4) statistical evaluation was conducted using several subsets. Also, different varieties of the same commodity have different protein and carbohydrate contents in the original, noncontaminated, state. In order to test the influence of these parameters on the robustness of the developed models, a sub-setting routine was implemented to choose specific combinations for testing. For example, only maize samples of the variety CRC infected with $F$. graminearum were chosen for detailed analysis before expanding the model by including the second SBL variety. Similarly, other fungal species were removed and added to test model robustness. For peanuts, less sample information was available and the dataset was subset by country.

Data pretreatment consisted of baseline correction, averaging, mean-centring, normalisation and calculation of the first derivative. A bagged decision tree classification of pretreated spectra yielded the best classification results for the chosen datasets and classification thresholds. PCA was carried out as a tool for observation of cluster formation, thus serving as a means for comparison, since PCA represents a wellestablished method in the scientific literature (see Table 2 for recent studies).

The bagged decision tree model was developed using the Statistics and Machine Learning Toolbox for Matlab 
Table 4. Summary of sample information for maize and peanuts.

\begin{tabular}{|c|c|c|c|c|c|c|c|}
\hline Commodity & $n$ & Toxin & Concentration range $\left(\mathrm{mg} \mathrm{kg}^{-1}\right)$ & Average $\left(\mathrm{mg} \mathrm{kg}^{-1}\right)$ & $\mathrm{SD}\left(\mathrm{mg} \mathrm{kg}^{-1}\right)$ & Fungi & Infection method \\
\hline \multicolumn{8}{|l|}{ Maize, $\mathrm{n}=184$} \\
\hline $\mathrm{CRC}$ & 90 & DON & $147-50,160$ & 8102 & 12,503 & $\begin{array}{l}\text { F. gram. } \\
\text { F. vert. } \\
\text { F. cul. }\end{array}$ & $\begin{array}{l}\text { Natural } \\
\text { Silk inj. } \\
\text { Toothpick }\end{array}$ \\
\hline SBL & 94 & DON & $<$ LOD-43,520 & 10,750 & 14,850 & $\begin{array}{l}\text { F. gram. } \\
\text { F. vert. } \\
\text { F. cul. }\end{array}$ & $\begin{array}{l}\text { Natural } \\
\text { Silk inj. } \\
\text { Toothpick }\end{array}$ \\
\hline \multicolumn{8}{|l|}{ Peanuts, $\mathrm{n}=92$} \\
\hline Burkina Faso & 35 & AFB1 & $<$ LOD-7960 & 615 & 1772 & n.a. & Natural \\
\hline Mozambique & 47 & AFB1 & $<$ LOD-10,624 & 708 & 1678 & n.a. & Natural \\
\hline Tanzania & 10 & AFB1 & $<10-98$ & 44 & 28 & n.a. & Natural \\
\hline
\end{tabular}

'fitensemble' routine (version R2015a) with 300 ensemble learning cycles. For each commodity, the trained classifier was then subjected to a cross-validation with 10 folds. Classification accuracy was calculated as: (1 classification loss) $\times 100$, thus reported as classification accuracy (\%). Classification loss was calculated as the loss averaged over all folds during cross-validation.

The use of cross-validation aimed at using the full sample variability provided by the dataset and conducting multiple validation runs with training and testing subsets to calculated classification accuracy. Thus we avoided a major drawback inherent with training and test validation (also called the 'split-sample approach'), which would have resulted in relatively few samples, 445 especially in the concentration range around the classification threshold. A consequence would have been a lack of representativity in the training and test sets, respectively. Cross-validation has been described as an 'extension of the split-sample method' (Steyerberg et al. 2001) since several random subsamples are selected as training sets for model development and then tested on the test sets, i.e., the remaining data (Refaeilzadeh et al. 2009). Also, in this study the training routine was performed at least five times to 455 investigate further model stability. Since the bagged decision tree method always starts with different starting conditions through bootstrap aggregation (Breiman 1996), results are expected to be slightly different. However, classification accuracy differences between 460 runs were always less than $0.6 \%$, thus serving as a further indicator of model stability.

\section{Results and discussion}

We have investigated the classification ability of a decision tree at $1750 \mu \mathrm{g} \mathrm{kg}^{-1}$ for deoxynivalenol in 465 maize and $8 \mu \mathrm{g} \mathrm{kg}^{-1}$ for aflatoxin $B_{1}$ in peanuts. These corresponded to regulatory limits set by the EU for unprocessed maize and peanuts destined for processing (Table 1). The limit for peanuts also includes shelled peanuts used here for FTIR measurements. For determination of the aflatoxin content, all the contamination is assumed to be on the edible part, as stated by Commission Regulation (EC) No. 165/ 2010. In order to study further the performance of the decision tree classifier, we also tested the data with a $500 \mu \mathrm{g} \mathrm{kg}^{-1}$ threshold for deoxynivalenol in maize. For comparison, corresponding PCA results were calculated.

Figures 2(a) and (b) provide the confusion matrix for decision tree classification results for maize. Among a total of 110 samples in the dataset, 17 samples with deoxynivalenol concentrations $<1750 \mu \mathrm{g} \mathrm{kg}^{-1}$ (first line) were correctly classified and 17 samples incorrectly classified. For samples with deoxynivalenol concentrations > $1750 \mu \mathrm{g} \mathrm{kg}^{-1}$ (second line), six samples were incorrectly classified and 70 samples correctly assigned as contaminated. Overall classification accuracy after cross-validation was $79 \%$, i.e., 87 out of 110 samples were correctly classified for the $1750 \mu \mathrm{g} \mathrm{kg}{ }^{-1}$ classifier. For the $500 \mu \mathrm{g} \mathrm{kg}^{-1}$ threshold in Figure 2(b), the results changed to three correct classifications and 15 incorrectly classified samples for concentrations $<500 \mu \mathrm{g} \mathrm{kg}^{-1}$. Only one sample was incorrectly classified as above the threshold, while 91 samples with concentrations $>500 \mu \mathrm{g} \mathrm{kg}^{-1}$ were correctly classified. In this case, overall classification accuracy was $85 \%$ after cross-validation (94 out of 110 samples). Score plots of principal components 1 and 2 show significant overlap between tentatively assigned clusters of samples with contamination above and below the threshold of $1750 \mu \mathrm{g} \mathrm{kg}^{-1}$ (Figure 3(a)). In an analogous fashion. Figures 3(b) and (c) provides decision tree and PCA results for peanut samples. Note that despite the much lower classification limit of $8 \mu \mathrm{g} \mathrm{kg}^{-1}$, model performance was in a similar range as maize with a classification accuracy of $77 \%$ for 92 peanut samples (Figure 3(b), 71 samples correctly classified). Again, a tentative cluster assignment for PCA calculations showed significant 


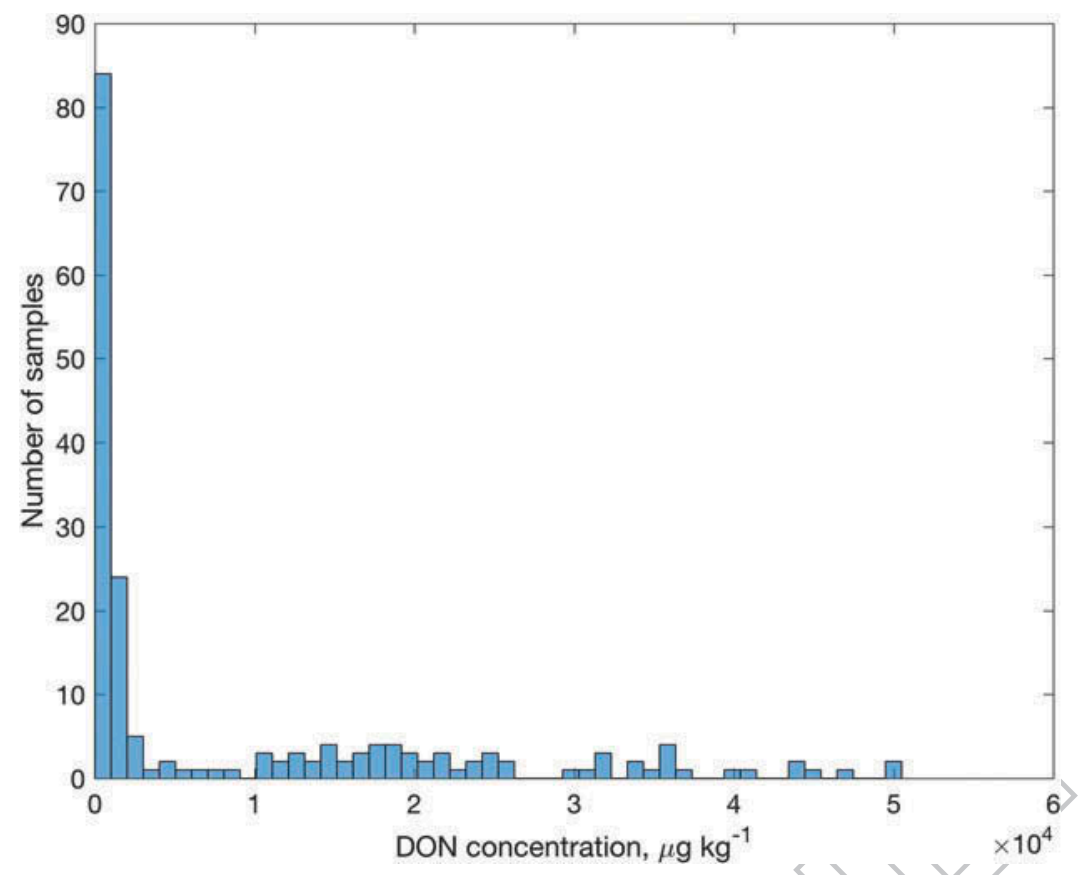

Figure 2(a). Histogram of (a) DON concentrations in maize samples investigated. $823 \times 617 \mathrm{~mm}(72 \times 72 \mathrm{DPI})$

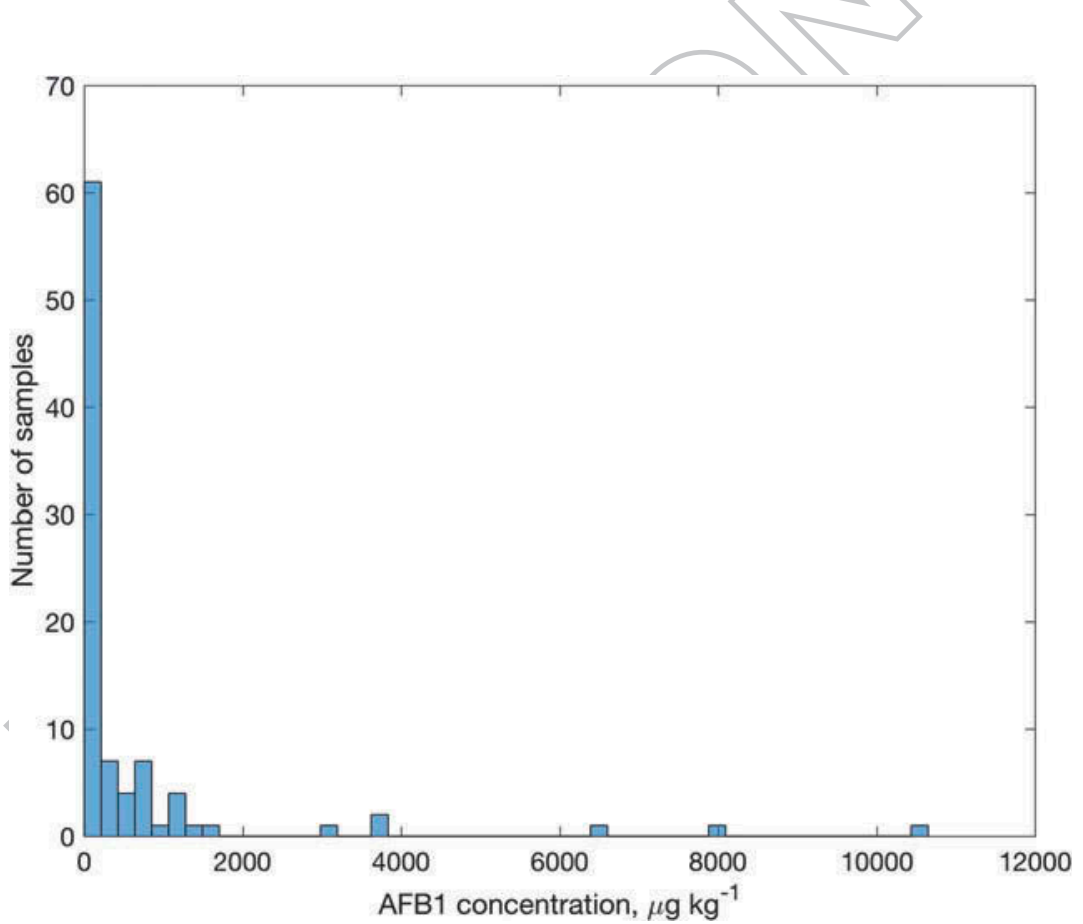

Figure 2(b). Histogram of (b) AFB1 concentrations in peanut samples investigated. $823 \times 617 \mathrm{~mm}(72 \times 72 \mathrm{DPI})$

overlap between clusters of samples with contamination above and below the threshold of $8 \mu \mathrm{g} \mathrm{kg}-1$ (Figure 3(c)). To our knowledge this is the first report of the classification of MIR spectra at these low concentrations using a decision tree method.

Both concentration limits for maize and peanuts used for classification above and below the chosen thresholds of $1750 \mu \mathrm{g} \mathrm{kg}^{-1}$ deoxynivalenol in maize to current EU regulatory limits. Final results, achieving a balance between classification accuracy and model stability (i.e., avoiding over-fitting), were obtained by thorough investigation of subsets based on available sample information. For maize samples, two different varieties (CRC and SBL) were available and separate models were created for both. While, for example, a CRC-only model resulted in a classification accuracy of 90\%, the addition of SBL samples increased the sample 


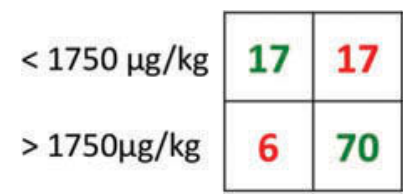

Figure 3(a). Classification results for maize samples with contamination above and below the threshold of $1750 \mu \mathrm{g} \mathrm{kg}^{-1}$; data labels provide deoxynivalenol concentration in $\mu \mathrm{g} \mathrm{kg}^{-1}$. The spectral window between 1800 and $800 \mathrm{~cm}^{-1}$ was used for chemometric modelling.

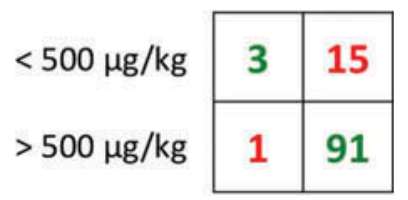

Figure 3(b). Classification results for maize samples with contamination above and below the threshold of $500 \mu \mathrm{g} \mathrm{kg}^{-1}$ using the decision tree method; data labels provide deoxynivalenol concentration in $\mu \mathrm{g} \mathrm{kg}^{-1}$. The spectral window between 1800 and $800 \mathrm{~cm}^{-1}$ was used for chemometric modelling.

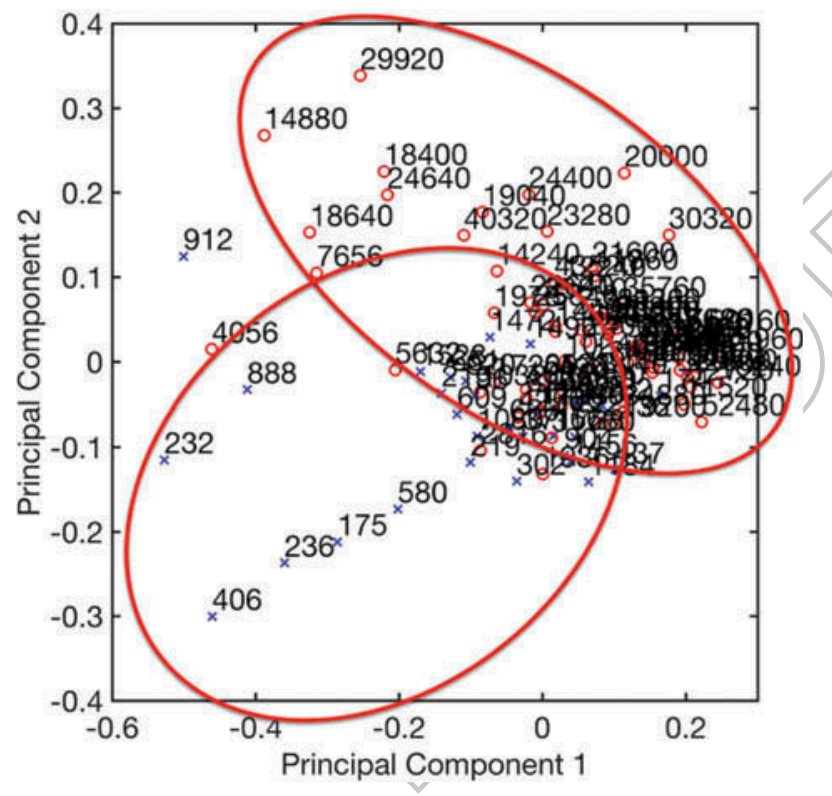

Figure 3(c). Classification results for maize samples employing PCA score plots for principal components 1 and 2 using the $1750 \mu \mathrm{g} \mathrm{kg}^{-1}$ classification threshold with tentative assignment of clusters; data labels provide deoxynivalenol concentration in $\mu \mathrm{gg}^{-1}$. The spectral window between 1800 and $800 \mathrm{~cm}^{-1}$ was used for chemometric modelling.

number from 72 to 110 , though there was degraded model performance (79\%) compared with single-variety subsets, but not to unacceptable levels. The latter is preferable since more samples can be used for modelling, thus contributing to increased model stability. As 530 a consequence, pooling of the two different available varieties was feasible, but we speculate that further investigation and model revalidation will be required for addition of other varieties.

A similar approach investigated the impact of different fungal species present in the sample in model performance. While the presence of $F$. graminearum and $F$. culmorum did not have an impact on model performance, samples contaminated with $F$. verticillioides decreased classification accuracy only to $73 \%$. Therefore, 74 samples infected with $F$. verticillioides were removed. A possible explanation could be that it has been reported that protein content alteration depends on the fungal species with which the commodity was infected (Boyacioğlu \& Hettiarachchy 1995). Since protein bands were part of the spectral window used for chemometric modelling, it was feasible that the presence of $F$. verticillioides negatively impacted classification accuracy. For peanuts, which were sampled from African markets, additional sample information such as variety and fungal contamination was unavailable. The presence of aflatoxins within a large proportion of these peanut samples indicates widespread contamination by Aspergillus species.

Seventeen out of 110 samples in maize were classified as false-positives by the decision tree model. Note, too, that the model errs on the safe side. Also, only three contaminated samples were classified as below the defined threshold of $1750 \mu \mathrm{g} \mathrm{kg}^{-1}$. This is in part due to the number of 23 low contamination samples compared with 87 contaminated ones. For peanuts, results of false-positives (10 out of 36 blanks; 28\%) and false-negatives (11 out of 56 contaminated; $20 \%$ ) were similar.

The concentration distribution of the target analyte in the model training set was important (Figures 4(a) and (b)). A large training set with a wide range of concentrations was essential for classification at the desired concentration level. Five out of 110 maize samples were close to the regulatory threshold with deoxynivalenol concentrations between 1500 and $2000 \mu \mathrm{g} \mathrm{kg}^{-1}, 32$ samples contained less than $1500 \mu \mathrm{g} \mathrm{kg}^{-1}$, and 73 samples more than $2000 \mu \mathrm{g} \mathrm{kg}^{-1}$. For peanuts, aflatoxin $B_{1}$ concentrations were between 4 and $12 \mu \mathrm{g} \mathrm{kg}^{-1}$ for seven samples; 34

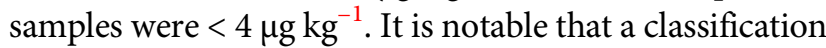
threshold of $500 \mu \mathrm{g} \mathrm{kg}^{-1}$ showed an improved classification accuracy of $85 \%$ after cross-validation. It has been pointed out that, ideally, the training set must be representative of the classes, and at the same time include the full range of variability for the class (Schowengerdt 2006). For the $500 \mu \mathrm{g} \mathrm{kg}^{-1}$ threshold the number of samples around the regulatory threshold with deoxynivalenol concentrations between 250 and $750 \mu \mathrm{g} \mathrm{kg}^{-1}$ was larger (12 out of 110 maize compared with five for the $1750 \mu \mathrm{g} \mathrm{kg}^{-1}$ threshold). Therefore, we assume that the variability 

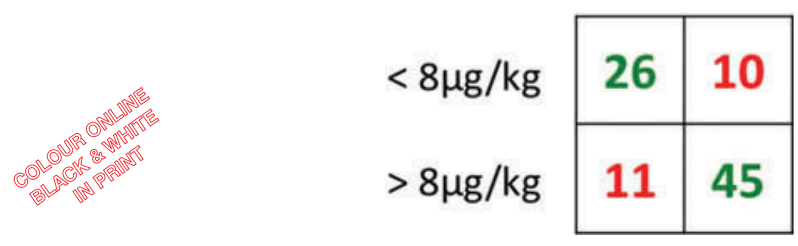

Figure 4(a). Classification results for peanut samples with contamination above and below the threshold of $8 \mu \mathrm{g} \mathrm{kg}^{-1}$; decision tree method; data labels provide aflatoxin $B_{1}$ concentration in $\mu \mathrm{g} \mathrm{kg}^{-1}$. The spectral window between 1800 and $800 \mathrm{~cm}^{-1}$ was used for chemometric modelling.

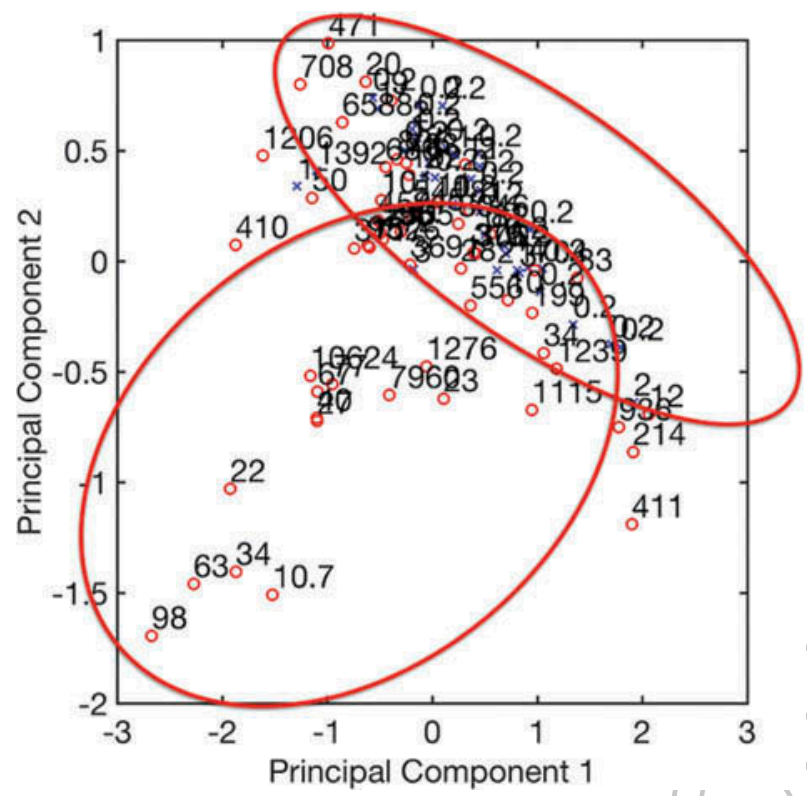

Figure 4(b). Classification results for peanut samples using PCA with tentative assignment of clusters; data labels provide aflatoxin $B_{1}$ concentration in $\mu \mathrm{g} \mathrm{kg}$. The spectral window between 1800 and $800 \mathrm{~cm}^{-1}$ was used for chemometric modelling.

around the threshold was better defined, resulting in a small performance increase. As a result, samples close to the regulatory threshold are important to be part of the training set in order to model small differences at the defined classifier concentration effectively. These should also cover the natural variability unrelated to fungal contamination in a representative fashion. Subsets with large concentration differences between samples, i.e., a large number of both blanks and highly contaminated samples without samples at the regulatory threshold, yielded impressive classification results for the training set. Poor results for samples with toxin levels at the regulatory threshold were observed, though.

Previous work (Table 2) attempting classification of deoxynivalenol contaminated samples in maize only employed a small number of 15 samples for classification, although a sample with a concentrations as low as
$309 \mu \mathrm{g} \mathrm{kg}^{-1}$ was classified as contaminated compared with true (i.e., below the reference method LOD) blanks (Kos et al. 2003). Regarding other matrices, the IR method presented by Abramovic et al. (2007) employed 17 samples (with three blanks < LOD) with a concentration range higher (i.e., $2.51-12.14 \mathrm{mg} \mathrm{kg}^{-1}$ ), making it impossible to attain the 1750 and $500 \mu \mathrm{g} \mathrm{kg}^{-1}$ classification thresholds presented here. Deoxynivalenol in wheat of a single genotype was determined. The most recent report by De Girolamo et al. (2014) using an linear discriminant analysis (LDA) classification model for wheat NIR data showed similar performance with $70-90 \%$ of samples correctly classified. The classifier for the smallest concentration was set to $<1000 \mu \mathrm{g} \mathrm{kg}^{-1}$. The sample set was large with 232 out of 464 samples used for model training. The authors noted an issue with the deoxynivalenol concentration distribution as a limiting factor for model stability for some of the multivariate methods tested. For peanuts this report is the first attempt to classify successfully aflatoxin $B_{1}$ at legal limits. Previous reports focused on quantification models making direct comparison difficult. Work by Fernandez-Ibanez et al. (2009) investigated 152 samples and defined samples with an aflatoxin $B_{1}$ concentration $<20 \mu \mathrm{g} \mathrm{kg}^{-1}$ as blanks. Qiang et al. (2014) investigated 82 rice samples using the same $20 \mu \mathrm{g} \mathrm{kg}$ ${ }^{1}$ lower limit for contaminated samples. Finally, with the same lower limit Lee et al. (2015) tested 232 maize samples with concentrations up to $1206 \mu \mathrm{g} \mathrm{kg}^{-1}$. This lower limit for contaminated samples were high and outside the EU legal limits compared with the $8 \mu \mathrm{g} \mathrm{kg}^{-1}$ used in the presented work.

The number of samples this study was an important step forward compared with previous work. Significant challenges remain to obtain well-defined, representative datasets with a suitable toxin concentration distribution. While this constitutes a considerable development effort, practical application is straightforward once the robustness of a model (e.g., for use with several varieties) has been established. Furthermore, 'self-learning' reference sets could be created by adding newly classified samples to the database after verification by LC-MS measurements. Thus, development of tailored models for well-defined sample sets, e.g., where variety and fungal contamination are known, becomes feasible.

\section{Conclusions}

The presented bagged decision tree method was able to classify maize samples at the 1750 and $500 \mu \mathrm{g} \mathrm{kg}^{-1}$ thresholds for deoxynivalenol with an accuracy of 
$79 \%$ and $85 \%$ respectively, and peanut samples for aflatoxin $B_{1}$ at $8 \mu \mathrm{g} \mathrm{kg}^{-1}$ with an accuracy of $77 \%$, thus demonstrating the method's capabilities to classify at the respective regulatory limits. For aflatoxin $B_{1}$ in peanuts, classification at these low concentration levels was achieved for the first time. Minimal sample preparation, speedy data acquisition and automatic data analysis provide a measurement platform suitable for use in the field, e.g., for testing at points of entry by importers and during storage and processing. It could be used to provide a fast overview of an imported batch after preliminary characterisation (i.e., determination of variety and fungal species present), and to provide a decision-making tool for importers, if a more thorough investigation by LC-MS is required. The method can be implemented without the need for highly qualified personnel on-site. The recent occurrence of afla670 toxins in European maize samples underlines the necessity and urgency for rapid, cheap mycotoxin detection methods available to food producers and importers as a changing climate potentially creates new challenges.

\section{Acknowledgements}

The authors thank Robert Taucher at Saatbau Linz, Austria, and Professor Akos Mesterhazy at the Cereal Research Centre, Szeged, Hungary, for providing the hybrids used in this study.

\section{Disclosure statement}

No potential conflict of interest was reported by the authors.

\section{Funding}

This work was supported by the European Union Seventh Framework Programme for Research, Technological Development and Demonstration [grant number 314018].

\section{ORCID}

Boris Mizaikoff (D) http://orcid.org/0000-0002-5583-7962

\section{References}

Abramovic B, Jajic I, Abramovic B, Cosic J, Juric V. 2007. Detection of deoxynivalenol in wheat by Fourier transform infrared spectroscopy. Acta Chim Slov. 54:859.

Al-Jowder O, Kemsley EK, Wilson RH. 1997. Mid-infrared spectroscopy and authenticity problems in selected meats: a feasibility study. Food Chem. 59:195-201.

Amiel C, Mariey L, Curk-Daubie MC, Pichon P, Travert J. 2000. Potentiality of Fourier transform infrared spectroscopy (FTIR) for discrimination and identification of dairy lactic acid bacteria. Le Lait. 80:445-459.
Boyacioğlu D, Hettiarachchy NS. 1995. Changes in some biochemical components of wheat grain that was infected with Fusarium graminearum. J Cereal Sci. 21:57-62.

Breiman L. 1996. Bagging predictors. Mach Learn. 24:123140.

Cerna M, Barros AS, Nunes A, Rocha SM, Delgadillo I, Copikova J, Coimbra MA. 2003. Use of FT-IR spectroscopy as a tool for the analysis of polysaccharide food additives. Carbohydr Polym. 51:383-389.

Chauhan R, Singh J, Sachdev T, Basu T, Malhotra BD. 2016. Recent advances in mycotoxins detection. Biosens Bioelectron. 81:532-545.

Choudhary AK, Kumari P. 2010. Management of mycotoxin contamination in pre-harvest and post-harvest crops: present status and future prospects. J Phytol. 2.

Chungu C, Mather D, Reid L, Hamilton R. 1996. Comparison of techniques for inoculating maize silk, kernel, and cob tissues with Fusarium graminearum. Plant Dis. 80:81-84.

Cozzolino D, Kwiatkowski M, Parker M, Cynkar W, Dambergs R, Gishen M, Herderich M. 2004. Prediction of phenolic compounds in red wine fermentations by visible and near infrared spectroscopy. Anal Chim Acta. 513:73-80.

Cozzolino D, Moron A. 2004. Exploring the use of near infrared reflectance spectroscopy (NIRS) to predict trace minerals in legumes. Anim Feed Sci Technol. 111:161173.

De Girolamo A, Cervellieri S, Visconti A, Pascale M. 2014. Rapid analysis of deoxynivalenol in durum wheat by FTNIR spectroscopy. Toxins. 6:3129-3143.

De Girolamo A, Lippolis V, Nordkvist E, Visconti A. 2009. Rapid and non-invasive analysis of deoxynivalenol in durum and common wheat by Fourier-transform near infrared (FT-NIR) spectroscopy. Food Addit Contam Part A. 26:907-917.

Draganova T, Daskalov P, Tsonev R. 2010. An approach for identifying of Fusarium infected maize grains by spectral analysis in the visible and near infrared region, SIMCA models, parametric and neural classifiers. Int J Bioautomation. 14:119-128.

Ertas N, Gonulalan Z, Yildirim Y, Karadal F. 2011. A survey of concentration of aflatoxin M1 in dairy products marketed in Turkey. Food Control. 22:1956-1959.

European Commission. 2002. Commission decision of 12 August 2002 implementing Council Directive 96/23/EC concerning the performance of analytical methods and the interpretation of results. Off J Eur Union. L221:9-36.

Fernandez-Ibanez V, Soldado A, Martinez-Fernandez A, De La Roza-Delgado B. 2009. Application of near infrared spectroscopy for rapid detection of aflatoxin b1 in maize and barley as analytical quality assessment. Food Chem. 113:629-634.

Fernández Pierna JA, Volery P, Besson R, Baeten V, Dardenne P. 2005. Classification of modified starches by Fourier transform infrared spectroscopy using support vector machines. J Agric Food Chem. 53:6581-6585.

Firrao G, Torelli E, Gobbi E, Raranciuc S, Bianchi G, Locci R. 2010. Prediction of milled maize fumonisin contamination by multispectral image analysis. J Cereal Sci. 52:327-330.

Groopman JD, Cain LG, Kensler TW, Harris CC. 1988. Aflatoxin exposure in human populations: measurements 
and relationship to cancer. CRC Crit Rev Toxicol. 19:113145.

IARC. 1993. Some naturally occurring substances: food items and constituents, heterocyclic aromatic amines and mycotoxins. Lyon: Working Group on the Evaluation of Carcinogenic Risks to Humans: International Agency for Research on Cancer.

IARC. 2016. Mycotoxin control in low- and middle-income countries.

Inon FA, Garrigues S, De La Guardia M. 2004. Nutritional parameters of commercially available milk samples by FTIR and chemometric techniques. Anal Chim Acta. 513:401-412.

Karoui R, Downey G, Blecker C. 2010. Mid-infrared spectroscopy coupled with chemometrics: a tool for the analysis of intact food systems and the exploration of their molecular structure-quality relationships - a review. Chem Rev. 110:6144-6168.

Kaya-Celiker H, Mallikarjunan PK, Kaaya A. 2016. Characterization of invasion of genus Aspergillus on peanut seeds using FTIR-PAS. Food Anal Methods. 9:105-113.

780 Kos G, Lohninger H, Krska R. 2003. Development of a method for the determination of Fusarium fungi on corn using mid-infrared spectroscopy with attenuated total reflection and chemometrics. Anal Chem. 75:1211-1217.

Lee K-M, Davis J, Herrman TJ, Murray SC, Deng Y. 2015. An empirical evaluation of three vibrational spectroscopic methods for detection of aflatoxins in maize. Food Chem. 173:629-639.

Lee KM, Herrman TJ. 2015. Determination and prediction of fumonisin contamination in maize by surface-enhanced Raman spectroscopy (SERS). Food Bioprocess Technol. 1.

Maertens K, Reyns P, De Baerdemaeker J. 2004. On-line measurement of grain quality with NIR technology. Trans ASAE. 47:1135-1140.

Malachová A, Beltran E, Sulyok M, Berthiller F, Krska R. 2015. Multi- toxin determination in food-the power of 'dilute and shoot' approaches in LC-MS-MS. LCGC Eur. 28.

Malachová A, Sulyok M, Beltran E, Berthiller F, Krska R. 2014. Optimization and validation of a quantitative liquid chromatography-tandem mass spectrometric method covering 295 bacterial and fungal metabolites including all regulated mycotoxins in four model food matrices. J Chromatogr A. 1362:145-156.

Mariey L, Signolle JP, Amiel C, Travert J. 2001. Discrimination, classification, identification of microorganisms using FTIR spectroscopy and chemometrics. Vib Spectrosc. 26:151-159.

Mark H, Workman J Jr. 2010. Chemometrics in spectroscopy. New York, NY: Academic Press.

Mauer LJ, Chernyshova AA, Hiatt A, Deering A, Davis R. 2009. Melamine detection in infant formula powder using near-and mid-infrared spectroscopy. J Agric Food Chem. 57:3974-3980.

McMullin D, Mizaikoff B, Krska R. 2015. Advancements in IR spectroscopic approaches for the determination of fungal derived contaminations in food crops. Anal Bioanal Chem. 407:653-660.

815 Mesterházy À. 1977. Reaction of winter wheat varieties to four Fusarium species. J Phytopathol. 90:104-112.

Miller JD. 2008. Mycotoxins in small grains and maize: old problems, new challenges. Food Addit Contam. 25:219-230.

Naes T, Isaksson T, Fearn T, Davies T. 2002. A user friendly guide to multivariate calibration and classification. Chichester, UK: NIR publications.
Pestka J. 2010. Toxicological mechanisms and potential health effects of deoxynivalenol and nivalenol. World Mycotoxin J. 3:323-347.

Prandini A, Tansini G, Sigolo S, Filippi L, Laporta M, Piva G. 2009. On the occurrence of aflatoxin $\mathrm{m} 1$ in milk and dairy products. Food Chem Toxicol. 47:984-991.

Qiang Z, Fuguo J, Chenghai L, Jingkun S, Xianzhe Z. 2014. Rapid detection of aflatoxin b1 in paddy rice as analytical quality assessment by near infrared spectroscopy. Int J Agric Biol Eng. 7:127.

Quinlan JR. 1986. Induction of decision trees. Mach Learn. 1:81-106.

Refaeilzadeh P, Tang L, Liu H. 2009. Cross-validation. In: Encyclopedia of database systems. Springer; p. 532-538.

Richard JL. 2007. Some major mycotoxins and their mycotoxicoses-an overview. Int J Food Microbiol. 119:3-10.

Rodrigues I, Handl J, Binder EM. 2011. Mycotoxin occurrence in commodities, feeds and feed ingredients sourced in the middle east and Africa. Food Addit Contam Part B. 4:168-179.

Schowengerdt RA. 2006. Remote sensing: models and methods for image processing. Academic Press.

Sirisomboon CD, Putthang R, Sirisomboon P. 2013. Application of near infrared spectroscopy to detect aflatoxigenic fungal contamination in rice. Food Control. 33:207-214.

Smela ME, Currier SS, Bailey EA, Essigmann JM. 2001. The chemistry and biology of aflatoxin b1: from mutational spectrometry to carcinogenesis. Carcinogenesis. 22:535-545.

Steyerberg EW, Harrell FE, Borsboom GJ, Eijkemans M, Vergouwe Y, Habbema JDF. 2001. Internal validation of predictive models: efficiency of some procedures for logistic regression analysis. J Clin Epidemiol. 54:774-781.

Swamy H, Smith TK, Karrow NA, Boermans HJ. 2004. Effects of feeding blends of grains naturally contaminated with Fusarium mycotoxins on growth and immunological parameters of broiler chickens. Poult Sci. 83:533-543.

Turner NW, Subrahmanyam S, Piletsky SA. 2009. Analytical methods for determination of mycotoxins: a review. Anal Chim Acta. 632:168-180.

Vardon P, McLaughlin C, Nardinelli C. 2003. Potential economic costs of mycotoxins in the United States. p. 139.

Vermeulen P, Pierna JAF, Egmond HPV, Dardenne P, Baeten V. 2012. Online detection and quantification of ergot bodies in cereals using near infrared hyperspectral imaging. Food Addit Contam Part A. 29:232-240.

Williams PC, Sobering DC. 1993. Comparison of commercial near infrared transmittance and reflectance instruments for analysis of whole grains and seeds. J Near Infrared Spectrosc. 1:25-32.

Williams PJ, Geladi P, Britz TJ, Manley M. 2012. Investigation of fungal development in maize kernels using NIR hyperspectral imaging and multivariate data analysis. J Cereal Sci. 55:272-278.

Wu F. 2007. Measuring the economic impacts of Fusarium toxins in animal feeds. Anim Feed Sci Technol. 137:363-374.

Young C, Kim S-S, Luzinova Y, Weida M, Arnone D, Takeuchi E, Day T, Mizaikoff B. 2009. External cavity widely tunable quantum cascade laser based hollow waveguide gas sensors for multianalyte detection. Sens Actuators B Chem. 140:24-28. 